\title{
ARBITRARY ORDER RECURSIVE SEQUENCES AND ASSOCIATED CONTINUED FRACTIONS
}

\author{
Anthony G. Shannon ${ }^{\mathrm{a}}$, Charles K. Cook ${ }^{\mathrm{b}}$, Rebecca A. Hillman ${ }^{\mathrm{c}}$ \\ ${ }^{a}$ Emeritus Professor, University of Technology Sydney, NSW 2007, Australia \& \\ Australian Institute of Musci, 1-55 Foveaux Street, Surry Hills, NSW 2010, Australia \\ ${ }^{b}$ Emeritus, University of South Carolina Sumter, Sumter, SC 29150, USA \\ ${ }^{\mathrm{c}}$ Associate Professor of Mathematics, University of South Carolina Sumter, Sumter, SC 29150, USA
}

\begin{abstract}
The essential idea in this paper it to generalize and synthesize some of the pioneering ideas of Bernstein, Lucas and Horadam on generalizations of basic and primordial Fibonacci numbers and their arbitrary order generalizations and their relation to generalized continued fractions with matrices as the unifying elements.
\end{abstract}

\section{Indexing terms/Keywords}

Extension field, Jacobi-Perron algorithm, recurrence relations, Fibonacci numbers, Lucas functions, Kronecker delta.

\section{Academic Discipline And Sub-Disciplines}

Mathematics: Number Theory

\section{SUBJECT CLASSIFICATION}

11B39, 11C20

\section{TYPE (METHOD/APPROACH)}

Properties of matrices associated with characteristic equations of arbitrary order difference equations are used to relate the associated sequences to a generalization of the continued fraction algorithm in the form of the Jacobi Perron Algorithm.

\section{INTRODUCTION}

The purpose of this paper is to demonstrate the existence of a one-to-one correspondence between a certain class of square matrices of arbitrary order and a related extension field. The elements of these matrices are eventually obtained from certain basic linear recursive sequences by generalizations of the ordinary continued fraction algorithm. This paper incorporates generalizations of Horadam's work [1] to show the connections among arbitrary order linear sequences. This accounts for the on-conventional use of superscripts.

Ward [2] established the existence of a one-to-one correspondence between a certain class of square matrices of order three (with elements from the field $F$ of the auxiliary polynomial $f_{1}(x)$ of a third order recurrence relation) and the field $F(\alpha)$ formed by the adjunction to $F$ of a root $\alpha$ of $f_{1}(x)=0$. Ward's results are generalized to sequences of arbitrary order in this paper and the elements of the class of square matrices of arbitrary order are related to certain basic linear recursive sequences (defined in Section 2) by means of a Jacobi-Perron algorithm for these elements in Section 4

\section{DEFINITIONS}

In the notation of this paper, Lucas studied the sequences $\left\{u_{0, n}^{(2)}\right\},\left\{u_{2, n}^{(2)}\right\}$ which satisfy the homogeneous second order linear recurrence relation

$$
u_{s, n}^{(2)}=P_{2,1} u_{s, n-1}^{(2)}-P_{2,2} u_{s, n-2}^{(2)}, s=0,2, n>2
$$

where the $P_{2, i}$ are arbitrary integers, and the sequences have initial conditions $u_{2,1}^{(2)}=0, u_{2,2}^{(2)}=1, u_{0,1}^{(2)}=2, u_{0,2}^{(2)}=\alpha_{2,1}+\alpha_{2,2}$, the sum of the roots of the auxiliary equation associated with (2.1), namely:

$$
0=x^{2}-P_{2,1} x+P_{2,2}
$$

$\left\{u_{0, n}^{(2)}\right\},\left\{u_{2, n}^{(2)}\right\}$ are Lucas' primordial and basic sequences, respectively, and in turn they are also generalizations of the Fibonacci and Lucas sequences, respectively. 
Horadam [3] developed properties of the more general sequence $\left\{w_{n}^{(2)}\right\}$ which satisfies the same recurrence relation (2.1) but has arbitrary initial conditions. Williams [4] extended Lucas' work both to arbitrary order and to the third order sequences, particularly $\left\{u_{s, n}^{(3)}\right\}, s=0,1,2, \mathrm{n}>2$, which satisfy the third order recurrence relation

$$
u_{s, n}^{(3)}=P_{3,1} u_{s, n-1}^{(3)}-P_{3,2} u_{s, n-2}^{(3)}+P_{3,3} u_{s, n-3}^{(3)}
$$

in which the $P_{3, i}$ are given complex numbers, and with appropriate initial conditions.

In this paper we consider a generalization of Horadam's sequences to arbitrary order $r$, we define $r$ basic sequences of order $r,\left\{u_{s, n}^{(r)}\right\}, s=1,2, \ldots, r$, by the recurrence relation

$$
u_{s, n}^{(r)}=\sum_{j=1}^{r}(-1)^{j+1} P_{r, j} u_{s, n-j}^{(r)}, n>r,
$$

in which the $P_{r, j}$ are arbitrary integers, and the initial terms, when $n=1,2, \ldots, r$, are given by

$$
u_{s, n}^{(r)}=\delta_{s, n},
$$

the Kronecker delta. The adjective "basic" is used by analogy with the corresponding third order sequence of Bell [5]. One of the basic sequences, $\left\{u_{r, n}^{(r)}\right\}$, is termed "fundamental" by analogy with Lucas' fundamental sequence of order 2. To correspond with the second order primordial sequence of Lucas [6,7] we define a primordial sequence of order $r$, $\left\{u_{0, n}^{(r)}\right\}$, which also satisfies the recurrence relation (2.4) but has initial terms

$$
u_{0, n}^{(r)}=\left\{\begin{array}{cc}
0, & n<1, \\
\sum_{j=1}^{r} \alpha_{r, j}^{n-1}, & 1 \leq n \leq r,
\end{array}\right.
$$

where the $\alpha_{r, j}$ are the roots (assumed distinct) of the characteristic equation associated with the recurrence relation (2.4):

$$
f(x) \equiv x^{r}-\sum_{j=1}^{r}(-1)^{j+1} P_{r, j} x^{r-j}=0,
$$

where

$$
f(x)=\prod_{i=1}^{r}\left(x-\alpha_{r, i}\right) .
$$

Generally in the literature only one basic second order sequence is mentioned, namely the fundamental one, but Gootherts [8] has shown the need for two basic second order sequences as well as the primordial sequence. The fundamental nature of the sequence, $\left\{u_{r, n}^{(r)}\right\}$, has been illustrated in effect by d'Ocagne (in Dickson [9]) who showed that any element $\left\{w_{n}^{(r)}\right\}$ of the set of all sequences of order $r, \Omega=\Omega\left(P_{r, 1}, P_{r, 2}, \ldots, P_{r, r}\right)$, which satisfy the recurrence relation (2.1), can be expressed in terms of the fundamental sequence and the initial terms of $\left\{w_{n}^{(r)}\right\}$ :

$$
w_{n}^{(r)}=\sum_{j=0}^{r-1} \sum_{k=j}^{r-1}(-1)^{k-j} P_{r, k-j} u_{r, n-k}^{(r)} w_{j}^{(r)}
$$

in which $P_{r, 0}=1$ for notational convenience. For example, when $r=2$,

$$
\begin{aligned}
w_{n}^{(2)} & =\sum_{j=0}^{1} w_{j}^{(2)} \sum_{k=j}^{1}(-1)^{k-j} P_{2, k-j} u_{2, n-k}^{(2)} \\
& =w_{0}^{(2)}\left(P_{2,0} u_{2, n}^{(2)}-P_{2,1} u_{2, n-1}^{(2)}\right)+w_{1}^{(2)} u_{2, n-1}^{(2)} \\
& =w_{1}^{(2)} u_{2, n-1}^{(2)}-P_{2,2} w_{0}^{(2)} u_{2, n-2}^{(2)}
\end{aligned}
$$

which agrees with Equation (3.14) of Horadam [1]. This is mentioned in order to emphasize that the results in this paper are quite generalizable.

Modifying Philippou [10] we now define $M_{n}^{(r)}$ as the square matrix of order $r$ : 


$$
M_{n}^{(r)}=\left[\begin{array}{cccc}
u_{1, n+1}^{(r)} & u_{2, n+1}^{(r)} & \ldots & u_{r, n+1}^{(r)} \\
u_{1, n+2}^{(r)} & u_{2, n+2}^{(r)} & \ldots & u_{r, n+2}^{(r)} \\
& \ldots & & \\
u_{1, n+r}^{(r)} & u_{2, n+r}^{(r)} & \ldots & u_{r, n+r}^{(r)}
\end{array}\right] .
$$

Thus $M_{0}^{(r)}=I_{r}$, the unit matrix of order $r$, and

$$
\begin{aligned}
M_{1}^{(r)} & =\left[\begin{array}{ccccc}
0 & 1 & 0 & \ldots & 0 \\
0 & 0 & 1 & \ldots & 0 \\
0 & 0 & 0 & \ldots & 1 \\
(-1)^{r+1} P_{r, r} & (-1)^{r} P_{r, r-1} & (-1)^{r-1} P_{r, r-2} & \ldots & P_{r, 1}
\end{array}\right] \\
& \equiv M,
\end{aligned}
$$

for notational convenience. This is similar to the $A$ matrix of [11] and is a generalization of the $Q$ (companion) matrix in the Fibonacci and Lucas number generators [12]. As in the second order $(r=2)$ case the following matrix properties are easily proved.

$$
\begin{aligned}
M_{n+1}^{(r)} & =M M_{n}^{(r)}=M^{n+1}, \\
M_{n}^{(r)} M_{m}^{(r)} & =M_{m}^{(r)} M_{n}^{(r)}=M_{m+n}^{(r)}, \\
M_{n+r}^{(r)} & =\sum_{j=1}^{r}(-1)^{j+1} P_{r, j} M_{n+r-j}^{(r)}, \\
M_{n-1}^{(r)} & =\sum_{j=1}^{(r)} u_{j, n}^{(r)} M_{j-1}^{(r)}, 1 \leq n \leq r .
\end{aligned}
$$

Equation (2.10) is a particular case of (2.11), and (2.12) follows easily from addition of matrices. It is also a generalization of a result used by Barakat [13]. The result (2.12) follows from addition of matrices. Equation (2.13) follows from (2.4) and is no less obvious because

$$
u_{j, n}^{(r)}=\delta_{j, n}, 1 \leq n \leq r
$$

For example,

$$
\begin{aligned}
M_{2}^{(r)} & =\sum_{j=1}^{r} u_{j, 3}^{(r)} M_{j-1}^{(r)} \\
& =\left\{\begin{array}{cc}
0 & j \neq 3, \\
M_{2}^{(r)} & j=3 .
\end{array}\right.
\end{aligned}
$$

\section{CHARACTERISTIC FUNCTION}

The result (2.10) shows that $M_{n}^{(r)}$ is a particular solution of the first order matrix difference equation

$$
\Omega_{n+1}=M \Omega_{n},
$$

and (2.12) shows that $M_{n}^{(r)}$ is a particular solution of the arbitrary order matrix difference equation

$$
\Omega_{n+1}=\sum_{j=1}^{r}(-1)^{j+1} P_{r, j} \Omega_{n-j} .
$$

We are now in a position to establish Theorem 1:

The characteristic function of the matrix $M_{n}^{(r)}$ is 


$$
\lambda^{r}-\sum_{m=1}^{r}(-1)^{m+1} s(r, n, m) \lambda^{r-m}
$$

where $s(r, n, m)$ is the symmetric function of the roots $\alpha_{r, j}$ of the auxiliary equation $f_{1}(x)=0$ taken $m$ at a time [14]:

$$
s(r, n, m)=\sum \alpha_{r, j_{1}}^{n} \alpha_{r, j_{2}}^{n} \ldots \alpha_{r, j_{m}}^{n} .
$$

Proof: As before we suppose that the roots are distinct. Then the matrix $M$ has $r$ linearly independent eigenvectors; that is, there are $r$ linearly independent eigenvectors; that is, there are $r$ distinct roots of the auxiliary equation

$$
0=\operatorname{det}(\lambda I-M)
$$

This follows because

$$
\begin{aligned}
\operatorname{det}(\lambda I-M)- & =\left|\begin{array}{ccccc}
\lambda & -1 & 0 & \ldots & 0 \\
0 & \lambda & -1 & \ldots & 0 \\
0 & 0 & \ldots & & \\
(-1)^{r} P_{r, r} & (-1)^{r-1} P_{r, r-1} & (-1)^{r-2} P_{r, r-2} & \ldots & \lambda-P_{r, 1}
\end{array}\right| \\
& =\lambda\left(\lambda^{r-1}-P_{r, 1} \lambda^{r-2}+\ldots\right)+(-1)^{r} P_{r, r} \\
& =\lambda^{r}-\sum_{j=1}^{r}(-1)^{j+1} P_{r, j} \lambda^{r-j} \\
& =f_{1}(\lambda),
\end{aligned}
$$

so that the eigenvalues of $M$ are the roots, $\alpha_{r, j}$, of the auxiliary equation associated with the recurrence relation. Tee [15] has used the symmetric functions for similar purposes with Vieta's Rule [16]. Thus there exists a non-singular matrix $X:$

$$
\begin{aligned}
M^{*} & =X^{-1} M X \\
& =\operatorname{diag}\left(\alpha_{r, 1}, \alpha_{r, 2}, \ldots, \alpha_{r, r}\right)
\end{aligned}
$$

Then

$$
\begin{aligned}
\left(M^{*}\right)^{2} & =\operatorname{diag}\left(\alpha_{r, 1}^{2}, \alpha_{r, 2}^{2}, \ldots, \alpha_{r, r}^{2}\right) \\
& =X^{-1} M^{2} X \\
& =X^{-1} M_{2}^{(r)} X
\end{aligned}
$$

from (2.10). Similarly,

$$
\begin{aligned}
\left(M^{*}\right)^{n} & =\operatorname{diag}\left(\alpha_{r, 1}^{n}, \alpha_{r, 2}^{n}, \ldots, \alpha_{r, r}^{n}\right) \\
& =X^{-1} M_{n}^{(r)} X
\end{aligned}
$$

which implies that the eigenvalues of $M_{n}^{(r)}$ are $\alpha_{r, j}^{n}, j=1,2, \ldots, r$, as required. The result then follows from the definition of the characteristic polynomial of the matrix $M_{n}^{(r)}$.

\section{PRIMORDIAL AND BASIC SEQUENCES AND CONTINUED FRACTIONS}

We can see in Table 1 some values for the first few terms of the second order $(r=2)$ primordial $(s=0)$ and basic $(s=1,2)$ sequences. This helps to visualize their roles in sequences of higher order than 2.

\begin{tabular}{|l|l|l|l|l|l|l|}
\hline $\boldsymbol{n}$ & $u_{0, n}^{(r)}$ & $u_{0, n}^{(2)}$ & $u_{1, n}^{(r)}$ & $u_{1, n}^{(2)}$ & $u_{2, n}^{(r)}$ & $u_{2, n}^{(2)}$ \\
\hline
\end{tabular}




\begin{tabular}{|c|l|l|l|l|l|c|}
\hline 1 & 2 & 2 & 1 & 1 & 0 & 0 \\
\hline 2 & $P_{2,1}$ & 1 & 0 & 0 & 1 & 1 \\
\hline 3 & $P_{2,1}^{2}-2 P_{2,2}$ & 3 & $-P_{2,2}$ & 1 & $P_{2,1}$ & 1 \\
\hline 4 & $P_{2,1}^{3}-3 P_{2,1} P_{2,2}$ & 4 & $-P_{2,1} P_{2,2}$ & 1 & $P_{2,1}^{2}-P_{2,2}$ & 2 \\
\hline 5 & $P_{2,1}^{4}-4 P_{2,1}^{2} P_{2,2}+2 P_{2,2}^{2}$ & 7 & $-P_{2,1}^{2} P_{2,2}+P_{2,2}^{2}$ & 2 & $P_{2,1}^{3}-2 P_{2,1} P_{2,2}$ & 3 \\
\hline
\end{tabular}

\section{Table 1: First five terms of the second order primordial and basic sequences}

It can be seen in the table that

$$
u_{1,3}^{(2)}+u_{2,4}^{(2)}=u_{0,3}^{(2)}
$$

More generally,

$$
u_{0, n+1}^{(r)}=\sum_{j=1}^{r} u_{j, n+j}^{(r)}
$$

which follows from the principle of mathematical induction or, more succinctly, from the symmetric function and the trace of $M_{n}^{(r)}$

$$
\begin{aligned}
u_{0, n+1}^{(r)} & =(-1)^{r} s(r, n, 1) \\
& =\operatorname{tr}\left(M_{n}^{(r)}\right) \\
& =\sum_{j=1}^{r} u_{j, n+j}^{(r)}
\end{aligned}
$$

We consider $u_{r, n}^{(r)}$ to be the fundamental basic sequence with the property that for its $r \times r$ matrix

$$
Q_{n}^{(r)}=\left[\begin{array}{cccc}
u_{r, n+1}^{(r)} & u_{r, n+2}^{(r)} & \ldots & u_{r, n+r}^{(r)} \\
u_{r, n+2}^{(r)} & u_{r, n+3}^{(r)} & \ldots & u_{r, n+r+1}^{(r)} \\
& \ldots & & \\
u_{r, n+r}^{(r)} & u_{r, n+r+1}^{(r)} & \ldots & u_{r, n+2 r-1}^{(r)}
\end{array}\right] .
$$

$Q_{n}^{(r)}$, like $M_{n}^{(r)}$, also satisfies (3.1) and (3.2). Not surprisingly then for the associated determinant we have

$$
\left|Q_{n}^{(r)}\right|=(-1)^{\frac{1}{2} r(r-1)} P_{r, r}^{n} .
$$

For example, from Table 1:

$$
\begin{aligned}
\left|Q_{1}^{(2)}\right| & =\left|\begin{array}{cc}
1 & P_{2,1} \\
P_{2,1} & P_{2,1}^{2}-P_{2,2}
\end{array}\right| \\
& =-P_{2,2} .
\end{aligned}
$$

By considering (3.1) and (3.2) we have that the most general solution is

$$
\Omega_{n}=M_{n}^{(r)} \Omega_{0}
$$

where the elements of the matrix $\Omega_{0}$ can be arbitrary. However, define a particular solution by putting 


$$
\Omega_{0}=\left[\begin{array}{cccc}
W_{0,0} & W_{1,0} & \ldots & W_{r-1,0} \\
W_{0,1} & W_{1,1} & \ldots & W_{r-1,1} \\
& & \ldots & \\
W_{0, r-1} & W_{1, r-1} & \ldots & W_{r-1, r-1}
\end{array}\right]
$$

where the $\{W j . j)$ are elements of $F$, so that

$$
\Omega_{m+n}=M_{n}^{(r)} \Omega_{m}
$$

from which subsequent properties immediately follow. Specifically, a necessary and sufficient condition for $\left\{\Omega_{n}\right\} \in \underline{M}$, the class of all matrices of the form $\sum_{j=0}^{r-1} W_{j} M^{j}$, is that the sequences $\left\{W_{m, n}\right\}, m=0,1, \ldots, r-2$, satisfy

$$
W_{m, n+j}=W_{m-1, n+j-1}+(-1)^{r-m+1} P_{r, r-m} W_{r-1, n+j-1}
$$

for $m=0,1, \ldots, r-2$, and $j=0, \pm 1, \pm 2, \ldots$. The proof follows symbolically from trivial but tedious matrix multiplication, but more importantly what follows from (4.5) is that if we extend it so that it is valid for $m=r-1$, then

$$
W_{r-1, n+1}=W_{r-j-1, n+j-1}-(-1)^{j} P_{r, j} W_{r-1, n},
$$

and in particular

$$
W_{r-1, n+1}=W_{r-2, n}+P_{r, 1} W_{r-1, n},
$$

If we next put

$$
a_{j-1}^{(n)}=\frac{W_{r-j, n}}{W_{r-1, n}}
$$

and

$$
b_{j}^{(n)}=(-1)^{j} P_{r, j}
$$

then

$$
\frac{W_{r-j, n+1} / W_{r-1, n}}{W_{r-1, n+1} / W_{r-1, n}}=\frac{W_{r-j-1, n} / W_{r-1, n}-(-1)^{j} P_{r, j}}{W_{r-2, n} / W_{r-1, n}-(-1) P_{r, 1}}
$$

becomes

$$
a_{j-1}^{(n+1)}=\frac{a_{j}^{(n)}-b_{j}^{(n)}}{a_{1}^{(n)}-b_{1}^{(n)}}
$$

if we set $a_{o}^{(n)}=1 \forall n$. Then let

$$
\left(a_{1}^{(k)}, a_{2}^{(k)}, \ldots, a_{r-1}^{(k)}\right)=a^{(k)} \in E_{r-1},
$$

the $(r-1)$-dimensional Euclidean space of $(r-1)$-tuples of real numbers $(r \geq 2)$ with $k$ a non-negative rational integer. Following Bernstein [17] we define the transformation $T: E_{r-1} \rightarrow E_{r-1}$ so that

$$
f\left(a^{(k)}\right)=b^{(k)}=\left(b_{1}^{(k)}, b_{2}^{(k)}, \ldots, b_{r-1}^{(k)}\right) \in E_{r-1}
$$

is any vector function on $E_{r-1}$ with values in $E_{r-1}$ such that

$$
a_{1}^{(k)} \neq b_{1}^{(k)} \text {. }
$$

Then call

$$
a^{(* k)} T=\frac{\left(a_{2}^{(k)}-b_{2}^{(k)}, a_{3}^{(k)}-b_{3}^{(k)}, \ldots, a_{r-1}^{(k)}-b_{r-1}^{(k)}, 1\right)}{a_{1}^{(k)}-b_{1}^{(k)}}
$$


a $T$-function. We further define a Jacobi-Perron Algorithm of the vector $a^{(0)}$ as a sequence $\left\{a^{(k)}\right\}$ of vectors in $E_{r-1}$ if there exists a $T$-transformation of $E_{r-1}$ into $E_{r-1}$ such that for every $k$

$$
a^{(k)} T=a^{(k+1)}
$$

which in effect shows the connection between the arbitrary order sequences and the JPA which are themselves generalizations of the Euclidean algorithm; this means that in the way Bernstein has defined them they are also generalizations of the ordinary continued fraction algorithm. This can be seen for $r=2$, when the elements of Table 1 are inserted in (4.8) [18].

\section{CONCLUDING COMMENTS}

Further related investigations for the interested reader could include:

(i) relating $\left|Q_{n}^{(r)}\right|$ to a vector solution of the linear Diophantine equations of the form

$$
1=\sum_{j=1}^{r} u_{r, n+j}^{(r)} x_{r}
$$

(ii) investigating the field properties of $M$ through (2.10), (2.12) and

$$
M^{r}=\sum_{j=1}^{r}(-1)^{j+1} P_{r, j} M^{r-j} .
$$

\section{References}

[1] Horadam, A.F. (1965) Basic Properties of a Certain Generalized Sequence of Numbers. The Fibonacci Quarterly. 3(4): 161-176.

[2] Ward, Morgan. (1931) The characteristic number of a sequence of integers satisfying a linear recurrence relation. Transactions of the American Mathematical Society. 33: 153-165.

[3] Horadam, A.F. (1965) Generating functions for powers of a certain generalized sequence of numbers. Duke Mathematical Journal. 32(3): 437-446.

[4] Williams, H.C. (1972) On a Generalization of the Lucas Functions. Acta Arithmetica. 20: 33-51.

[5] Bell, E.T. (1924) Notes on Recurring Series of the Third Order. Tôhoku Mathematical Journal. 24: 168-184.

[6] Kuhapatanakul, Kantaphon. (2015) The Lucas p-matrix. International Journal of Mathematical Education in Science and Technology. 46 (8): 1228-1234.

[7] Lucas, Edouard. (1878) Théorie des fonctions numériques simplement périodiques. American Journal of Mathematics. 1: 184-240, 289-321.

[8] Gootherts, G.W. (1968) A Lucas Algebra constructed from Fibonacci Sequences, Part I: Fundamentals and Polynomial Interpretations. The Fibonacci Quarterly. 6: 35-43.

[9] Dickson, L.E. (1952) History of the Theory of Numbers. Volume I. New York: Chelsea, Ch.XVII.

[10] Philippou, Andreas N. (1983) A Note on the Fibonacci Sequence of Order K and the Multinomial Coefficients. The Fibonacci Quarterly. 21(1): 82-86.

[11] Latushkin, Yaroslav, Vladimir Ushakov. (2009) A representation of recurrent sequences by previous terms. Congressus Numerantium. 194: 159-166.

[12] Brand, L. (1964) The Companion Matrix and Its Properties. American Mathematical Monthly. 71(6): 629-634.

[13] Barakat, Richard. (1964) The matrix operator $e^{X}$ and the Lucas polynomials. Journal of Mathematics and Physics. 43: 332-335.

[14] Macmahon, Percy A. (1915) Combinatory Analysis. Volume I. Cambridge: Cambridge University Press.

[15] Tee, Garry J. (1994) Prime powers of zeros of monic polynomials with integer coefficients. The Fibonacci Quarterly. 32(3): 277-283.

[16] Funkhouser, H. Gray. (1930) A short account of the history of symmetric functions of roots of equations. American Mathematical Monthly. 37(7): 357-365.

[17] Bernstein, Leon. (1971) The Jacobi-Perron Algorithm: Its Theory and Application. Lecture Notes in Mathematics, 207. Berlin: Springer Verlag. 


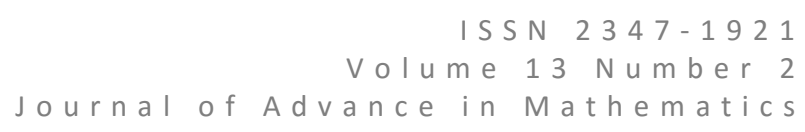

[18] Shannon, A.G. (1976) Pellian equations and continued fractions. New Zealand Mathematics Magazine. 13(3): 113115.

\section{Authors' biographies with Photos}
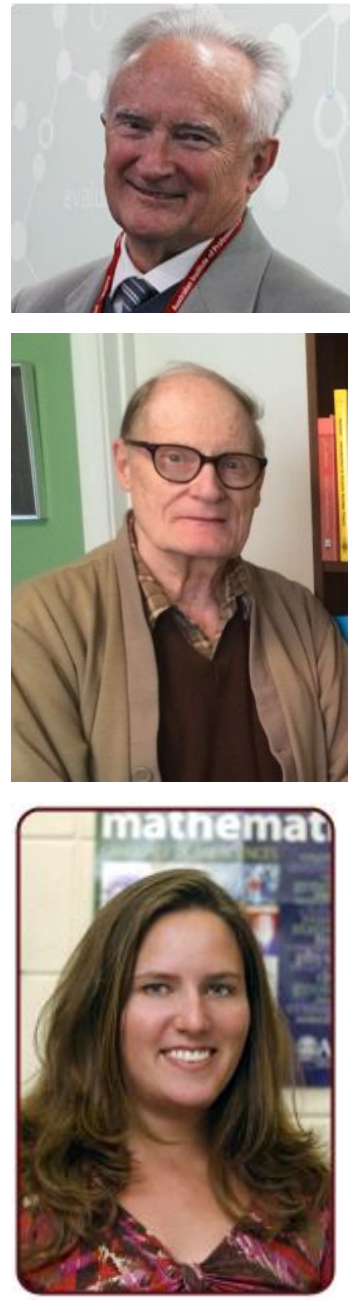

Professor A. G. (Tony) Shannon AM is an Emeritus Professor of the University of Technology, Sydney, where he was Foundation Dean of the UTS Graduate Research School and Professor of Applied Mathematics. He is currently Deputy Chancellor of the University of Notre Dame Australia, and Director of Aacdemic Studies at the Australian Institute of Music. He holds the doctoral degrees of Ph.D., Ed.D. and D.Sc. He is co-author of numerous books and articles in medicine, mathematics and education. He enjoys reading, walking, theatre, number theory, watching rugby, and thoroughbred racing.

Professor Charles K. Cook is a distinguished emeritus professor of mathematics of the University of South Carolina Sumter, Sumter, SC. USA. His primary research interests lie in Fibonacci and related sequential studies.

He holds a BEd from Chicago Teacher's College, an MS in mathematics from De Paul University and a PhD from Purdue University. His professional organizations include membership in AMS, AMA and The Fibonacci Association. He enjoys theater and mysteries, particularly those involving Sherlock Holmes; and belongs to two Baker Street Irregulars scion societies.

Rebecca A. Hillman (Becky) is an Associate Professor of Mathematics at The University of South Carolina Sumter. She earned her Ph.D. in Mathematics from North Carolina State University in 2005. She is co-author of many articles in mathematics and worked on a grant in the field of Math Education. Her research interests are in number theory (especially Fibonacci numbers), math education, and algebraic topology.

Dr. Hillman has a passion for teaching undergraduate mathematics and offers many of the math courses for the Elementary Education Bachelor's Degree through USC Upstate at Sumter. She is dedicated to reducing the perceived fear of mathematics, which typically begins at a young age and continues well into ones college career. She believes math is fun; but also enjoys vacationing, the beach, professional and college football, and nascar racing. 